\title{
A DUALITY THEOREM FOR AN ARBITRARY OPERATOR
}

\section{ERRETT BISHOP}

1. Introduction. This paper studies the spectral theory of an operator (or bounded linear transformation) on a Banach space $B$. Although it would be possible to modify the results to apply to operators on non-reflexive spaces, for simplicity only reflexive spaces are considered. The distinctive feature of the treatment is that we are primarily interested in the spectral properties of a completely arbitrary operator. That is, we seek a spectral theory which will be valid independently of any of the usual restrictions (such as normality or complete continuity). It is, of course, not to be expected, in view of many known counter examples, that such a theory will even approach in power the spectral theory of a Hermitian or normal operator on a Hilbert space. In fact, it is surprising that a spectral theory for an arbitrary operator exists at all. The results obtained here are incomplete, but it seems likely that any spectral theory which is valid for an arbitrary operator will be closely related to the theory developed here. It is interesting that certain known results in spectral theory, which imply the spectral theorem for a Hermitian or a unitary operator, can be obtained from the theory which we develop for an arbitrary operator. The principal ideas of this paper are developments of ideas which appeared in rudimentary form in unpublished portions of the author's University of Chicago doctoral dissertation.

The program of the paper is as follows. Instead of considering one type of spectral theory, we consider four. Since the content of these theories is that a certain duality exists between an operator and its adjoint, and since the term "spectral theory" is somewhat deceptive, we shall employ the term "duality theory" in its stead. The duality theories are not all of comparable strength. The weakest, type 4, will be shown to be valid for an arbitrary operator. This will be the principal result. This having been shown, it will then be possible to show that certain of the stronger and more conventional duality theories are satisfied provided that the operator is subject to appropriate restrictions. The duality theories will concern the existence and properties of spectral manifolds. Two types of spectral manifold are needed. The first type of spectral manifold, the strong type, is defined as one might expect, but it is the introduction of the second or weak type of spectral manifold that permits the construction of a theory which will apply to an arbitrary operator.

The two types of spectral manifold and the four types of duality

Received July 18, 1958. 
theory will be defined in $\S 2$, and certain elementary consequences of the definitions will be obtained. In $\S 3$ the principal result, that an arbitrary operator admits a duality theory of type 4, will be derived. The proof will entail a preliminary investigation of certain Banach spaces of analytic functions. In $\S 4$, the concluding section, conditions will be given under which the stronger duality theories obtain. In particular, conditions on the rate of growth of the norm $\left\|(T-z I)^{-1}\right\|$ of the resolvent will be given which imply the existence of a strong type of spectral theory. Such rate of growth conditions have been studied by Lorch [4] and Wolf [7], but the treatment given here is much more general and the methods are distinct.

2. Definitions and preliminaries. It can be seen that the definition given below of a strong spectral manifold coincides with the usual definition if the operator is Hermitian or if, more generally, the operator can be written in the form $\int \lambda d E(\lambda)$, in the sense of Lorch [4] or Dunford [2].

DEFINITION 1. Let $T$ be an operator on a reflexive Banach space $B$. Let $F$ be a closed subset of the complex plane. We define the strong spectral manifold $M(F, T)$ to be the closure of the set of all vectors $x$ in $B$ which have the property that there exists an analytic function $f$ on the complement $-F$ of $F$ with values in $B$ such that $(T-z I) f(z)=x$ for all $z$ in $-F$.

It will be seen that the strong spectral manifolds are not sufficient to give an adequate duality theory for an arbitrary operator. (What is meant by an adequate duality theory will become clear later.) We therefore define the second type of spectral manifold.

DEFINITION 2. Let $T$ be an operator on a reflexive Banach space $B$. Let $F$ be a closed subset of the complex plane. We define the weak spectral manifold $N(F, T)$ to be the set of all vectors $x$ in $B$ which have the property that for each $\varepsilon>0$ there exists an analytic function $f$ on the complement $-F$ of $F$ with values in $B$ such that

$$
\|(T-z I) f(z)-x\|<\varepsilon \text { for all } z \text { in }-F \text {. }
$$

It is clear from the definition that $N(F, T)$ is closed and that $M(F, T)$ is a subspace of $N(F, T)$. Examples can be given to show that $M(F, T)$ can actually be a proper subspace of $N(F, T)$; however, it can be show that $M(F, T)=N(F, T)$ if $T$ is a normal operator on a Hilbert space. It is also clear that $M\left(F_{1}, T\right) \subset M\left(F_{2}, T\right)$ and $N\left(F_{1}, T\right)$ $\subset N\left(F_{2}, T\right)$ if $F_{1} \subset F_{2}$. Also, $M(\sigma(T), T)=N(\sigma(T), T)=B$, where $\sigma(T)$ denotes the spectrum of $T$.

The task of investigating the spectral manifolds $M(F, T)$ and $N(F, T)$ 
now presents itself. An obvious possibility is to attempt to prove, for instance, that $M\left(F_{1}, T\right)$ and $M\left(F_{2}, T\right)$ span $M\left(F_{1} \cup F_{2}, T\right)$. Although this is the case if $T$ acts on a finite-dimensional space or if $T$ is a normal operator on a Hilbert space, it is not true in general. A much more promising possibility for investigation is suggested by the fact that if $B$ is finite-dimensional and if $F_{1}$ and $F_{2}$ are disjoint closed sets with $F_{1} \cup F_{2} \supset \sigma(T)$ then the subspace $M\left(F_{2}, T^{*}\right)$ of $B^{*}$ is the annihilator $M\left(F_{1}, T\right)^{\perp}$ of the subspace $M\left(F_{1}, T\right)$ of $B$. This is easily seen from the theory of operators on finite-dimensional spaces. By means of the theory of integration around subsets of $\sigma(T)$ which are both open and closed, which has been developed by Lorch and is given in Hille [3], it is not difficult to show that this statement remains valid without the restriction that $B$ be finite-dimensional. In case it is not possible to decompose $\sigma(T)$ into disjoint closed sets, the essence of the statement can nevertheless be recaptured. This is done by means of the following definition.

Definition 3. An operator $T$ on a reflexive Banach space $B$ will be said to admit a duality theory of type 1 if $M\left(F_{1}, T\right)^{\perp} \supset M\left(F_{2}, T^{*}\right)$ whenever $F_{1}$ and $F_{2}$ are compact and disjoint and if $M\left(\bar{G}_{1}, T\right)^{\perp} \subset M\left(\bar{G}_{2}, T^{*}\right)$ whenever $\bar{G}_{1}$ and $\bar{G}_{2}$ are the closures of open sets $G_{1}$ and $G_{2}$ which together cover the complex plane.

It is clear that $T$ and $T^{*}$ play symmetric roles in this definition because $B$ is reflexive and because the inclusions $M\left(F_{1}, T\right)^{\perp} \supset M\left(F_{2}, T^{*}\right)$ and $M\left(\bar{G}_{1}, T\right)^{\perp} \subset M\left(\bar{G}_{2}, T^{*}\right)$ are equivalent respectively to the inclusions

$$
M\left(F_{2}, T^{*}\right)^{\perp} \supset M\left(F_{1}, T\right) \text { and } M\left(\bar{G}_{2}, T^{*}\right)^{\perp} \subset M\left(\bar{G}_{1}, T\right) .
$$

Later we shall see that not every operator admits a duality theory of type 1. More precisely, there exist an operator $T$ and open sets $G_{1}$ and $G_{2}$ which cover the complex plane such that $M\left(\bar{G}_{1}, T\right)^{\perp}$ is not a subset of $M\left(\bar{G}_{2}, T^{*}\right)$. Since it will be seen that the inclusion $M\left(F_{1}, T\right)^{\perp}$ $\supset M\left(F_{2}, T^{*}\right)$ is always valid, it is a case of the strong spectral manifolds being too small. This is what was meant above when it was asserted that the strong spectral manifolds are not sufficient to give an adequate duality theory for an arbitrary operator, and this is the reason for consideration of the weak spectral manifolds. Conditions on an operator which imply that it admits a duality theory of type 1 will be obtained in $\$ 4$. Since the duality theory of type 1 does not have much surface resemblance to the usual spectral theories, we now define duality theories of types 2 and 3 which bear a closer surface resemblance to the usual spectral theories, but which will be seen to be intimately related to the duality theory of type 1 . Some of the ideas occurring in the development of these duality theories are closely related to ideas which occur in [1]. 
Definition 4. The operator $T$ on the reflexive Banach space $B$ will be said to admit a duality theory of type 2 if $M\left(\bar{G}_{1}, T\right), \cdots, M\left(\bar{G}_{n}, T\right)$ span $B$ whenever $G_{1}, \cdots, G_{n}$ are open sets which cover the complex plane.

If $T$ is an operator and $M$ is a subspace of $B$ which is invariant under $T$, the restriction of $T$ to $M$ will be denoted by $T / M$.

Definition 5. Let $T$ be an operator on a reflexive Banach space $B$ such that for arbitrary open sets $G_{1}, \cdots, G_{n}$ which cover the complex plane there exist subspaces $M_{1}, \cdots, M_{n}$ which span $B$ and are invariant under $T$ and have the property that $\sigma\left(T / M_{i}\right) \subset \bar{G}_{i}$. Then $T$ is said to admit a duality theory of type 3 .

It was shown by Lorch [4] that $T$ admits a duality theory of type 3 , in our terminology, if (1) $\sigma(T) \subset\{z:|z|=1\}$ and (2) there exists a constant $K$ such that $\left\|T^{n}\right\| \leqq K$ for all positive and negative integers $n$. This work has been generalized by various authors. In $\$ 4$ general conditions will be obtained which imply that $T$ admits a duality theory of type 3. We now define the fourth type of duality theory.

DEFinition 6. Let $T$ be an operator on a reflexive Banach space such that the inclusions $M\left(F_{1}, T\right)^{\perp} \supset N\left(F_{2}, T^{*}\right), N\left(F_{1}, T\right)^{\perp} \supset M\left(F_{2}, T^{*}\right)$, $M\left(\bar{G}_{1}, T\right)^{\perp} \subset N\left(\bar{G}_{2}, T^{*}\right)$, and $N\left(\bar{G}_{1}, T\right)^{\perp} \subset M\left(\bar{G}_{2}, T^{*}\right)$ are valid for arbitrary disjoint compact sets $F_{1}$ and $F_{2}$ and arbitrary open sets $G_{1}$ and $G_{2}$ which cover the complex plane. Then $T$ is said to admit a duality theory of type 4.

It is clear that $T$ and $T^{*}$ play symmetric roles in this definition. Although this definition seems awkeward, it is justified by the nontrivial theorem, to be proved below, that every operator on a reflexive Banach space admits a duality theory of type 4 . This fact makes it plausible that there exists a third type of spectral manifold $L(F, T)$, intermediate between $M(F, T)$ and $N(F, T)$, which is self-dual in the sense that $L\left(F_{1}, T\right)^{\perp} \supset L\left(F_{2}, T^{*}\right)$ and $L\left(\bar{G}_{1}, T\right)^{\perp} \subset L\left(\bar{G}_{2}, T\right)$ whenever $F_{1}$ and $F_{2}$ are disjoint and compact and $G_{1}$ and $G_{2}$ are open and cover the complex plane. The author has not succeeded in formulating a conjecture as to how such intermediate spectral manifolds might be obtained.

3. Duality theory of type 4. Since the definitions of the spectral manifold involve vector-valued analytic functions, it will be necessary to make a preliminary study of spaces composed of such functions. Let $B$ be a Banach space with dual $B^{*}$. Let $U_{1}$ be an unbounded open subset of the complex plane whose boundary $C$ consists of a finite number of disjoint closed rectifiable Jordan curves. The set $U_{1}$ will be called an unbounded simple open set. Let $U_{2}$ be the complement 
of $U_{1} \cup C$, so that $U_{2}$ is a bounded open set whose boundary is $C$. The set $U_{2}$ will be called a bounded simple open set, and $U_{1}$ and $U_{2}$ will be called complementary simple open sets. Let $W_{1}$ be the set of all analytic functions from $U_{1}$ to $B$ which vanish at infinity, and let $W_{2}$ consist of all analytic functions from $U_{2}$ to $B^{*}$. We topologize $W_{1}$ and $W_{2}$ by the topology of uniform convergence on compact subsets of $U_{1}$ and $U_{2}$ respectively. It is clear that $W_{1}$ and $W_{2}$ are locally convex linear topological spaces. Let $V_{1}$ consist of those functions in $W_{1}$ which can be extended to be continuous on $U_{1} \cup C$, and let $V_{2}$ consist of those functions in $W_{2}$ which can be extended to be continuous on $U_{2} \cup C$. If $f \in V_{1}$ we define $\|f\|=\sup \left\{\|f(z)\|: z \in U_{1}\right\}$, and if $g \in V_{2}$ we define $\|g\|=\sup \left\{\|g(z)\|: z \in U_{2}\right\}$. The Banach space $V_{1}$ is called the Banach space of analytic functions from $U_{1}$ to $B$ which have continuous boundary values, and $V_{2}$ is called the Banach space of analytic functions from $U_{2}$ to $B^{*}$ which have continuous boundary values.

Before proceeding, we state the Cauchy integral formula in a form suitable to our purposes: there exists a unique orientation on $C$, called the canonical orientation, such that

$$
g\left(z_{0}\right)=\frac{1}{2 \pi i} \int_{0} g(z)\left(z-z_{0}\right)^{-1} d z
$$

whenever $z_{0} \in U_{2}$ and $g$ is an analytic function on some neighborhood of $U_{2} \cup C$, and such that

$$
f\left(z_{0}\right)=-\frac{1}{2 \pi i} \int_{C} f(z)\left(z-z_{0}\right)^{-1} d z
$$

whenever $z_{0} \in U_{1}$ and $f$ is an analytic function on some neighborhood of $U_{1} \cup C$ which vanishes at infinity.

For $z$ in $U_{1}, z_{0}$ in $U_{2}$, and $x$ in $B$ we define $\alpha\left(x, z_{0}, z\right)=\left(z-z_{0}\right)^{-1} x$. For each $x$ in $B$ and each $z_{0}$ in $U_{2}$, the element $\alpha\left(x, z_{0}, \cdot\right)$ will be called an elementary element of $V_{1}$. The Banach space $V$, called the restricted Banach space of analytic functions from $U_{1}$ to $B$ which have continuous boundary values, is defined to be that subspace of $V_{1}$ which is spanned by the elementary elements of $V_{1}$.

If $f \in V_{1}$ and $g \in V_{2}$, we define

$$
\langle f, g\rangle=\frac{1}{2 \pi i} \int_{0}\langle f(z), g(z)\rangle d z,
$$

where $C$ is given the canonical orientation and where we have extended $f$ and $g$ to be continuous on $C$. It is clear that this defines a jointly continuous bilinear functional between $V_{1}$ and $V_{2}$. 
LEMMA 1. Let $V$ be the restricted Banach space of analytic functions from an unbounded simple open set $U_{1}$ to a Banach space $B$ which have continuous boundary values. Let $V_{2}$ and $W_{2}$ be defined as above. Then there exists a linear subset $Y$ of $W_{2}$ and a norm on $Y$ such that

(1) $Y$ is a Banach space.

(2) $V_{2} \subset Y$.

(3) The inclusion mappings $V_{2} \rightarrow Y$ and $Y \rightarrow W_{2}$ are continuous.

(4) The inner product between $V$ and $V_{2}$ can be extended to an inner product between $V$ and $Y$ in such a way that $Y$ becomes isometrically isomorphic to $V^{*}$.

Proof. Let $\rho$ be an arbitrary element of $V^{*}$. For each $x$ in $B$ and $z_{0}$ in $U_{2}$ define $\Gamma\left(x, z_{0}\right)=\Gamma_{\varphi}\left(x, z_{0}\right)=\phi\left(\alpha\left(x, z_{0}, \cdot\right)\right)$. For $z_{0}$ fixed, it follows that $\Gamma\left(\cdot, z_{0}\right)$ is a continuous linear functional on $B$, because convergence of $\left\{x_{n}\right\}$ to $x$ in $B$ implies convergence of $\left\{\alpha\left(x_{n}, z_{0}, \cdot\right)\right\}$ to $\alpha\left(x, z_{0}, \cdot\right)$ in $V_{1}$, which in turn implies convergence of $\left\{\phi\left(\alpha\left(x_{n}, z_{0}, \cdot\right)\right)\right\}=$ $\left\{\Gamma\left(x_{n}, z_{0}\right)\right\}$ to $\varphi\left(\alpha\left(x, z_{0}, \cdot\right)\right)=\Gamma\left(x, z_{0}\right)$. Thus $\Gamma\left(\cdot, z_{0}\right) \in B^{*}$ for each $z_{0}$ in $U_{2}$. We now prove that $I^{\top} \in W_{2}$. By this we mean that the mapping $z_{0} \rightarrow \Gamma\left(\cdot, z_{0}\right)$ is an analytic function from $U_{2}$ to $B^{*}$. It is sufficient to show that this mapping is weakly analytic, i.e., to show that $z_{0} \rightarrow \Gamma\left(x, z_{0}\right)$ is an analytic function on $U_{2}$ for each $x$ in $B$ (see Hille [3], page 53). To this end we consider the difference quotient

$$
\begin{aligned}
& {\left[\Gamma\left(x, z_{1}\right)-\Gamma\left(x, z_{0}\right)\right]\left(z_{1}-z_{0}\right)^{-1}} \\
& \quad=\mathscr{P}\left\{\left[\alpha\left(x, z_{1}, \cdot\right)-\alpha\left(x, z_{0}, \cdot\right)\right]\left(z_{1}-z_{0}\right)^{-1}\right\} .
\end{aligned}
$$

As $z_{1} \rightarrow z_{0}$, the element $\left[\alpha\left(x, z_{1}, \cdot\right)-\alpha\left(x, z_{0}, \cdot\right)\right]\left(z_{1}-z_{0}\right)^{-1}$ converges in $V$ to $\beta\left(x, z_{0}, \cdot\right)$, where $\beta\left(x, z_{0}, z\right)$ is defined by $\beta\left(x, z_{0}, z\right)=\left(z-z_{0}\right)^{-2} x$ for $x$ $\mathrm{i}_{\mathrm{n}} B, z_{0}$ in $U_{2}$, and $z$ in $U_{1}$. The difference quotient in question therefore converges to $\mathcal{P}\left(\beta\left(x, z_{0}, \cdot\right)\right)$ as $z_{1} \rightarrow z_{0}$. It follows that $\Gamma \in W_{2}$. We have thus defined a linear mapping $\varphi \rightarrow I^{\prime}{ }_{\varphi}$ of $V^{*}$ into $W_{2}$. Let $Y$ be the range of this mapping, so that $Y \subset W_{2}$. If $\Gamma_{\varphi}=0$, then $\varphi\left(\alpha\left(x, z_{0}, \cdot\right)\right)=0$ for all $x$ in $B$ and all $z_{0}$ in $W_{2}$, which implies that $\mathcal{P}$ vanishes on all elementary elements of $V_{1}$. Since the elementary elements span $V$, it follows that $\varphi=0$. Thus the mapping $\phi \rightarrow \Gamma_{\varphi}$ is a one-to-one mapping of $V^{*}$ onto $Y$. Hence $Y$ is a Banach space under the norm $\left\|I_{\varphi}\right\|=$ $\|\varphi\|$. Consider a net $\left\{\Gamma_{\varphi_{n}}\right\}$ of elements of $Y$ which converges to $\Gamma_{\varphi}$ in $Y$, so that $\left\{\varphi_{n}\right\}$ converges to $\varphi$ in $V^{*}$. We wish to show that $\left\{\Gamma_{\varphi_{n}}\right\}$ converges to $\Gamma_{\varphi}$ in $W_{2}$, or that $\left\{\Gamma_{\varphi_{n}}\right\}$ converges uniformly to $\Gamma_{\varphi}$ on each compact subset $F$ of $U_{2}$. This is equivalent to the convergence of the net $\left\{\rho_{n}\left(\alpha\left(x, z_{0}, \cdot\right)\right)\right\}$ to $\varphi\left(\alpha\left(x, z_{0}, \cdot\right)\right)$ uniformly for $\|x\| \leqq 1$ and $z_{0}$ in $F$. This in turn follows from the fact that $\left\{\alpha\left(x, z_{0}, \cdot\right):\|x\| \leqq 1, z_{0} \in F\right\}$ is

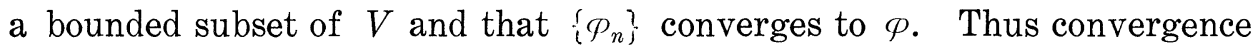
of a net $\left\{\Gamma_{\varphi_{n}}\right\}$ in $Y$ implies convergence of the net in $W_{2}$ to the same 
limit, so that the inclusion map $Y \rightarrow W_{2}$ is continuous.

To prove $V_{2} \subset Y$, consider $g$ in $V_{2}$ and define the continuous linear functional $\phi$ on $V$ by $\phi(f)=\langle f, g\rangle$, where the inner product between $V_{1}$ and $V_{2}$ was defined above. Thus $\mathcal{P} \in V^{*}$, so that $\Gamma_{\varphi} \in Y$. Also,

$$
\begin{aligned}
\Gamma_{\varphi}\left(x, z_{0}\right) & =\varphi\left(\alpha\left(x, z_{0}, \cdot\right)\right)=\left\langle\alpha\left(x, z_{0}, \cdot\right), g\right\rangle \\
& =\frac{1}{2 \pi i} \int_{C}\left\langle\alpha\left(x, z_{0}, z\right), g(z)\right\rangle d z \\
& =\frac{1}{2 \pi i} \int_{C}\left(z-z_{0}\right)^{-1}\langle x, g(z)\rangle d z=\left\langle x, g\left(z_{0}\right)\right\rangle .
\end{aligned}
$$

Thus $\Gamma_{\varphi}=g$. It follows that $V_{2} \subset Y$.

We define the inner product between $V$ and $Y$ by $\left\langle f, \Gamma_{\varphi}\right\rangle=\varphi\left(f^{\prime}\right)$, so that $Y$ becomes isometrically isomorphic to $V^{*}$. Since we have seen in the preceding paragraph that $\varphi(f)=\langle f, g\rangle$ for each $g=I_{\varphi}^{\prime}$ in $V_{2}$, it follows that this inner product extends the inner product between $V$ and $V_{2}$.

It remains to show that the inclusion map $V_{2} \rightarrow Y$ is continuous. Let $\left\{g_{n}\right\}$ converge to $g$ in $V_{2}$. Define $\mathscr{P}_{n}$ in $V^{*}$ by $\mathscr{P}_{n}(f)=\left\langle f, g_{n}\right\rangle$ for all $f$ in $V$, so that $g_{n}=\Gamma_{\varphi_{n}}$ by the above. To show that $\left\{g_{n}\right\}$ converges to $g$ in $Y$ we must show that $\left\{\varphi_{n}\right\}$ converges to $\phi$ in $V^{*}$. This follows from the joint continuity of the inner product between $V$ and $V_{2}$. This completes the proof of the lemma.

We shall identify the space $Y$ of Lemma 1 with $V^{*}$, so that $V^{*}$ will be thought of as a subset of $W_{2}$. Define the bounded linear mapping $\tau$ of $V$ into $B$ by $\tau f=\lim _{z \rightarrow \infty} z f(z)$. Then the adjoint mapping $\tau^{*}$ of $B^{*}$ into $V^{*}$ is defined by taking $\tau^{*} u$ to be that function of $V_{2}$ whose value at each point of $U_{2}$ is $u$, i.e., by setting $\left(\tau^{*} u\right)(z)=u$ for all $z$ in $U_{2}$. This is seen by the following computation:

$$
\begin{aligned}
\langle\tau f, u\rangle=\lim _{z \rightarrow \infty} z\langle f(z), u\rangle & =\lim _{z \rightarrow \infty} z\left[-\frac{1}{2 \pi i} \int_{0}(t-z)^{-1}\langle f(t), u\rangle d t\right] \\
& =\frac{1}{2 \pi i} \int_{0}\langle f(t), u\rangle d t .
\end{aligned}
$$

The mapping $\tau$ is onto, because $\tau \alpha\left(x, z_{0}, \cdot\right)=x$ for all $x$ in $B$ and all $z_{0}$ in $U_{2}$, as a simple computation shows.

In addition to the above objects, we now consider an operator $T$ on $B$. We define the operator $H$ on $V$ by $(H f)(z)=(T-z I) f(z)+\tau f$ for all $z$ in $U_{1}$. In order to obtain a formula for the adjoint operator $H^{*}$ on $V^{*}$, consider an elementary element $f=\alpha\left(x, z_{0}, \cdot\right)$ of $V$ and an element $g$ of $V^{*}$. We consider $g$ as an element of $W_{2}$, and let $\mathcal{P}$ be the element of $V^{*}$ which corresponds to $g$. Then $\left\langle x, g\left(z_{0}\right)\right\rangle=\mathcal{P}\left(\alpha\left(x, z_{0}, \cdot\right)\right)=$ $\phi(f)$, by the definition of $g$. Also, $\langle f, g\rangle=\mathcal{P}(f)$ when $g$ is interpreted 
as the element $\mathcal{P}$ of $V^{*}$. Thus $\langle f, g\rangle=\left\langle x, g\left(z_{0}\right)\right\rangle$.

Similarly, $\left\langle f, H^{*} g\right\rangle=\left\langle x,\left(H^{*} g\right)\left(z_{0}\right)\right\rangle$ and $\left\langle\alpha\left(T x, z_{0}, \cdot\right), g\right\rangle=\left\langle T x, g\left(z_{0}\right)\right\rangle$. Thus

$$
\begin{aligned}
& (H f)(z)=(T-z I)\left(z-z_{0}\right)^{-1} x+x \\
& \quad=\alpha\left(T x, z_{0}, z\right)-z\left(z-z_{0}\right)^{-1} x+x=\alpha\left(T x, z_{0}, z\right)-z_{0}\left(z-z_{0}\right)^{-1} x .
\end{aligned}
$$

Therefore $H f=\alpha\left(T x, z_{0}, \cdot\right)-z_{0} \alpha\left(x, z_{0}, \cdot\right)$. Thus

$$
\begin{aligned}
\left\langle x,\left(H^{*} g\right)\left(z_{0}\right)\right\rangle & =\left\langle f, H^{*} g\right\rangle=\langle H f, g\rangle \\
& =\left\langle T x, g\left(z_{0}\right)\right\rangle-z_{0}\left\langle x, g\left(z_{0}\right)\right\rangle=\left\langle x,\left(T^{*}-z_{0} I\right) g\left(z_{0}\right)\right\rangle .
\end{aligned}
$$

Since this is true for all $x$ in $B$, it follows that the adjoint operator is given by the formula $\left(H^{*} g\right)\left(z_{0}\right)=\left(T^{*}-z_{0} I\right) g\left(z_{0}\right)$ for all $z_{0}$ in $U_{2}$.

Of the many possible norms for $V \times V$, we consider only those norms obtained by defining

$$
\left\|\left(f_{1}, f_{2}\right)\right\|=\left\|\left(f_{1}, f_{2}\right)\right\|_{\eta}=\left(\eta\left\|f_{1}\right\|^{2}+\left\|f_{2}\right\|^{2}\right)^{1 / 2},
$$

where $\eta$ is an arbitrary positive constant. Then the norm on $V^{*} \times V^{*}$ will be given by $\left\|\left(g_{1}, g_{2}\right)\right\|=\left(\eta^{-1}\left\|g_{1}\right\|^{2}+\left\|g_{2}\right\|^{2}\right)^{1 / 2}$. We define an operator $K$ on $V \times V$ by $K\left(f_{1}, f_{2}\right)=\left(f_{1}, H f_{1}-f_{2}\right)$. A simple computation shows that $K^{2}=I$ and that $K^{*}$ is the operator on $V^{*} \times V^{*}$ defined by $K^{*}\left(g_{1}, g_{2}\right)=\left(H^{*} g_{2}+g_{1},-g_{2}\right)$. Since $K^{2}=I$ it has an inverse and therefore the norm [ ] on $V \times V$ defined by $[f]=\|K f\|$ is equivalent to the norm \|\| . Similarly, the norm [ ] on $V^{*} \times V^{*}$ defined by $[g]=\left\|K^{*} g\right\|$ is equivalent to the norm \|\| . Also, for each $g$ in $V^{*} \times V^{*}$ we have

$$
\begin{aligned}
{[g] } & =\left\|K^{*} g\right\|=\sup \left\{\left|\left\langle f, K^{*} g\right\rangle\right|:\|f\|=1\right\} \\
& =\sup \{|\langle K f, g\rangle|:\|f\|=1\} \\
& =\sup \{|\langle K f, g\rangle|:\|K(K f)\|=1\}=\sup \{|\langle h, g\rangle|:[h]=1\} .
\end{aligned}
$$

Thus the norm [ ] on $V^{*} \times V^{*}$ corresponds to the norm [ ] on $V \times V$.

In the proof of Theorem 1 we shall need the following simple lemmas. They correspond to well-known results, but we sketch their proofs for the sake of completeness and in order to have them in exactly the form they are needed.

LEMMA 2. Let $A$ be a bounded linear transformation which maps a Banach space $B_{1}$ homeomorphically into a Banach space $B_{2}$. Then the norm [ ] on $B_{1}^{*}$ defined by $[u]=\inf \left\{\|v\|: v \in B_{2}^{*}, A^{*} v=u\right\}$ corresponds to the norm [ ] on $B_{1}$ defined by $[x]=\|A x\|$.

Proof. Consider $u$ in $B_{1}^{*}$. By the Hahn-Banach theorem, there exists $v$ in $B_{2}^{*}$ such that $\langle x, u\rangle=\langle A x, v\rangle$ for all $x$ in $B_{1}$ and such that $\|v\|=\sup \left\{|\langle A x, v\rangle|: x \in B_{1},\|A x\| \leqq 1\right\}$. Thus $A^{*} v=u$. If also $A^{*} v_{1}=u$, 
then $\left\langle A x, v_{1}\right\rangle=\langle A x, v\rangle$ for all $x$ in $B_{1}$, so that

$$
\left\|v_{1}\right\| \geqq \sup \left\{\left|\left\langle A x, v_{1}\right\rangle\right|: x \in B_{1},\|A x\| \leqq 1\right\}=\|v\| .
$$

Thus

$$
\begin{aligned}
{[u] } & =\|v\|=\sup \left\{|\langle A x, v\rangle|: x \in B_{1},\|A x\| \leqq 1\right\} \\
& =\sup \left\{|\langle x, u\rangle|: x \in B_{1},[x] \leqq 1\right\}
\end{aligned}
$$

as was to be proved.

Lemma 3. Let $A$ be a bounded linear transformation of a Banach space $B_{2}$ onto a Banach space $B_{1}$. Then the norm on $B_{1}^{*}$ defined by $[u]=\left\|A^{*} u\right\|$ corresponds to the norm on $B_{1}$ defined by $[x]=\inf \{\|y\|:$ $A y=x$, and the norms [ ] and \|| II on $B_{1}$ are equivalent.

Proof. It is easily seen that [ ] is a norm on $B_{1}$ and that $\|x\| \leqq$ $\|A\|[x]$ for all $x$ in $B_{1}$. Let $\left\{x_{n}\right\}$ be a Cauchy sequence of elements in $B_{1}$ relative to the norm [ ]. We may assume, by passing to a subsequence if necessary, that $\left[x_{n}-x_{n+1}\right]<2^{-n}$. Thus we can inductively find $y_{n}$ in $B_{2}$ with $A y_{n}=x_{n}$ and $\left\|y_{n}-y_{n+1}\right\|<2^{-n}$. Thus $\left\{y_{n}\right\}$ converges to a vector $y$ in $B_{2}$. Thus $\left\{x_{n}\right\}$ converges to $x=A y$ relative to the norm [ ] on $B_{1}$. Thus $B_{1}$ is complete under the norm [ ]. By the closed graph theorem, it follows that the norms \|\| and [] on $B_{1}$ are equivalent. Also, if $u \in B_{1}^{*}$ then $[u]=\left\|A^{*} u\right\|=\sup \left\{\left|\left\langle y, A^{*} u\right\rangle\right|: y \in B_{2},\|y\| \leqq 1\right\}=$ $\sup \left\{|\langle A y, u\rangle|: y \in B_{2},\|y\| \leqq 1\right\}=\sup \left\{|\langle x, u\rangle|: x \in B_{1},[x] \leqq 1\right\}$, as was to be proved.

The following theorem is the guiding result of this paper. The other theorems will be derived as consequences.

THEOREM 1. Let $T$ be an operator on a Banach space $B$. Let $U_{1}$ be an unbounded simple open set, and let $V$ be the restricted Banach space of analytic functions from $U_{1}$ to $B$. Let $\eta$ be positive. For $x$ in $B$ define

$$
\{x\}=\{x\}_{\eta}=\inf \left\{\left(\eta\|f\|^{2}+\|H f\|^{2}\right)^{1 / 2}: f \in V, \tau f=x\right\}_{j} .
$$

For $u$ in $B^{*}$ define

$$
\{u\}=\{u\}_{\eta}=\inf \left\{\left(\|g\|^{2}+\eta^{-1}\left\|H^{*} g-\tau^{*} u\right\|^{2}\right)^{1 / 2}: g \in V^{*}\right\} .
$$

Then the function \{\} on $B$ is a norm which is equivalent to the norm II II and the function \{\} on $B^{*}$ is the norm on $B^{*}$ which corresponds to the norm \{\} on $B$.

Proof. The map $(u, g) \rightarrow\left(\tau^{*} u, g\right)$ of $B^{*} \times V^{*}$ into $V^{*} \times V^{*}$ is the 
adjoint of the map $\left(f_{1}, f_{2}\right) \rightarrow\left(\tau f_{1}, f_{2}\right)$ of $V \times V$ onto $B \times V$. It follows from Lemma 3 that the norm on $B^{*} \times V^{*}$ defined by $[(u, g)]=\left[\left(\tau^{*} u, g\right)\right]$ corresponds to the norm on $B \times V$ defined by $[(x, f)]=\inf \left\{\left[\left(f_{0}, f\right)\right]\right.$ : $\left.\tau f_{0}=x\right\}$, and that the norms [ ] and \|\| on $B \times V$ are equivalent. Now the map $(u, g) \rightarrow u$ of $B^{*} \times V^{*}$ onto $B^{*}$ is the adjoint of the map $x \rightarrow(x, 0)$ of $B$ into $B \times V$. It follows from Lemma 2 that the norm defined on $B^{*}$ by $[u]=\inf \left\{[(u, g)]: g \in V^{*}\right\}$ corresponds to the norm defined on $B$ by $[x]=[(x, 0)]$, and that the norms [ ] and \|\| on $B$ are equivalent. But

$$
\begin{aligned}
{[x] } & =\inf \{[(f, 0)]: \tau f=x\}=\inf \{\|K(f, 0)\|: \tau f=x\} \\
& =\inf \{\|(f, H f)\|: \tau f=x\} \\
& =\inf \left\{\left(\gamma\|f\|^{2}+\|H f\|^{2}\right)^{1 / 2}: \tau f=x\right\}=\{x\} .
\end{aligned}
$$

Also,

$$
\begin{aligned}
{[u] } & =\inf \left\{\left[\left(\tau^{*} u, g\right)\right]: g \in V^{*}\right\}=\inf \left\{\left\|K^{*}\left(\tau^{*} u, g\right)\right\|: g \in V^{*}\right\} \\
& =\inf \left\{\left\|\left(H^{*} g+\tau^{*} u,-g\right)\right\|: g \in V^{*}\right\} \\
& =\inf \left\{\left\|\left(H^{*} g-\tau^{*} u, g\right)\right\|: g \in V^{*}\right\} \\
& =\inf \left\{\left(\eta^{-1}\left\|H^{*} g-\tau^{*} u\right\|^{2}+\|g\|^{2}\right)^{1 / 2}: g \in V^{*}\right\}=\{u\} .
\end{aligned}
$$

This proves Theorem 1.

Of the two constituents, $\eta^{1 / 2}\|f\|$ and $\|H f\|$, which enter into the norm $\{x\}=\inf \left\{\left(\gamma\|f\|^{2}+\|H f\|^{2}\right)^{1 / 2}: \tau f=x\right\}$ on $B$, imprecisely speaking the second constituent

$$
\|H f\|=\sup \left\{\|(T-z I) f(z)+x\|: z \in U_{1}\right\}
$$

measures the degree of approximation to which one can solve the resolvent equation $(T-z I) f(z)=x$ on $U_{1}$, and the first $\eta^{\frac{1}{2}}\|f\|$ measures the size of the approximate solution. A similar comment can be made for the norm \{\} on $B^{*}$. Thus Theorem 1, which states that the norms correspond, establishes a relationship between approximate solutions to the resolvent equation for $T$ on the set $U_{1}$ and approximate solutions to the resolvent equation for $T^{*}$ on the set $U_{2}$. In view of the fact that the spectral manifolds were defined in terms of approximate solutions to the resolvent equation, it is not surprising that Theorem 1 should contain information pertinent to the duality theories defined above. Actually, the duality theory which we obtain as a consequence of Theorem 1 will be less precise than Theorem 1 itself. It therefore seems likely that a complete duality theory for an arbitrary operator will be based upon some version of Theorem 1 itself rather than upon the duality theory which we now proceed to derive from Theorem 1. It would have been possible to derive Theorem 1 in more generality by considering possibilities other than $V$ for the Banach space of analytic functions from $U_{1}$ to 
$B$, but this was not necessary for the applications to be made.

Corollary. Let the subset $N$ of $B$ consist of all $x$ such that for each $\varepsilon>0$ there exists $f$ in $V$ with $\|H f\|<\varepsilon$ and $\tau f=x$. Let the subspace $M$ of $B^{*}$ be the closure of the set $M_{0}$ of all $u$ in $B^{*}$ such that there exists $g$ in $V^{*}$ with $H^{*} g=\tau^{*} u$. Then $N$ is a subspace and $M^{\perp}=N$.

Proof. We first show that $N=\left\{x:\{x\}_{n} \rightarrow 0\right.$ as $\left.\eta \rightarrow 0\right\}$. If $x \in N$ for each $\varepsilon>0$ there exists $f$ in $V$ with $\tau f=x$ and $\|H f\|<\varepsilon$. Thus $\{x\}_{\eta} \leqq\left(\eta\|f\|^{2}+\varepsilon^{2}\right)^{1 / 2} \rightarrow \varepsilon$ as $\eta \rightarrow 0$. Since $\varepsilon$ is arbitrary it follows that $\{x\}_{\eta} \rightarrow 0$ as $\eta \rightarrow 0$. Conversely if $\{x\}_{\eta} \rightarrow 0$ as $\eta \rightarrow 0$ then for each $\varepsilon>0$ there exists $\eta>0$ such that $\{x\}_{\eta}<\varepsilon$, which implies that there exists $f$ in $V$ with $\tau f=x$ and $\left(\eta\|f\|^{2}+\|H f\|^{2}\right)^{1 / 2}<\varepsilon$. Thus $\|H f\|<\varepsilon$, so that $x \in N$.

We next show that $M_{0}=\{u:\{u\} \eta$ is bounded for $\eta>0\}$. If $u \in M_{0}$, then there exists $g$ in $V^{*}$ with $H^{*} g=\tau^{*} u$. Therefore

$$
\{u\}_{\eta} \leqq\left(\eta^{-1}\left\|H^{*} g-\tau^{*} u\right\|^{2}+\|g\|^{2}\right)^{1 / 2}=\|g\|
$$

is bounded for $\eta>0$. Conversely, assume now that $\{u\}_{\eta}<c$ for all positive $\eta$. Then for each positive integer $n$ there exists $g_{n}$ in $V^{*}$ with $\left(n\left\|H^{*} g_{n}-\tau^{*} u\right\|^{2}+\left\|g_{n}\right\|^{2}\right)<c^{2}$. Thus $\left\|g_{n}\right\|<c$. Since the unit sphere of $V^{*}$ is compact in the weak star topology, there exists a cluster point $g$ of $\left\{g_{n}\right\}$ in the weak star topology. Therefore $H^{*} g$ is a cluster point of $\left\{H^{*} g_{n}\right\}$ in the weak star topology. Since $\left\|H^{*} g_{n}-\tau^{*} u\right\|^{2} \leqq c^{2} n^{-1}$, it follows that $H^{*} g=\tau^{*} u$. Therefore $u \in M_{0}$.

If $x \in N$ and $u \in M_{0}$, then $|\langle x, u\rangle| \leqq\{x\}_{\eta}\{u\}_{\eta} \rightarrow 0$ as $\eta \rightarrow 0$. Therefore $N$ and $M_{0}$ are orthogonal. If $x$ is not in $N$, then $\{x\}_{\eta}$ does not converge to 0 as $\eta \rightarrow 0$, so that there exists a sequence $\left\{\eta_{n}\right\}$ of positive constants converging to 0 and a constant $c>0$ such that $\{x\}_{\eta_{n}}>c$ for all $n$. It follows that there exists a sequence $\left\{u_{n}\right\}$ of elements in $B^{*}$ with $\left\{u_{n}\right\}_{\eta_{n}}<1$ and $\left\langle x, u_{n}\right\rangle>c$. We can thus find a sequence $\left\{g_{n}\right\}$ of elements in $V^{*}$ with

$$
\eta_{i n}^{-1}\left\|H^{*} g_{n}-\tau^{*} u_{n}\right\|^{2}+\left\|g_{n}\right\|^{2}<1 .
$$

As before, there exists a cluster point $(g, u)$ of the sequence $\left\{\left(g_{n}, u_{n}\right)\right\}$ in the weak star topology of $V^{*} \times B^{*}$. It follows, as before, that $H^{*} g=\tau^{*} u$. Thus $u \in M_{0}$. Since also $\langle x, u\rangle \geqq c$, the vector $x$ is not in $M_{0}^{\perp}$. Thus $M_{0}^{\perp}=N$. It follows that $N$ is a subspace and that $M^{\perp}=N$, as was to be proved.

As an easy consequence of this corollary, we obtain the following fundamental theorem. 
THeORem 2. Every operator $T$ on a reflexive Banach space $B$ admits a duality theory of type 4.

Proof. Let $F_{1}$ and $F_{2}$ be disjoint compact sets. Then there exists an unbounded simple open set $U_{1}$, whose complementary simple open set will be called $U_{2}$, such that $F_{2} \subset U_{1}$ and $F_{1} \subset U_{2}$. Define $N$ as in the above corollary. To see that $N\left(F_{1}, T\right) \subset N$, take $x$ in $N\left(F_{1}, T\right)$ and take $\varepsilon>0$. Let $z_{0}$ be any point in $U_{2}$ and let $\inf \left\{\left|z-z_{0}\right|: z \in U_{1}\right\}=c>0$. By the definition of $N\left(F_{1}, T\right)$, there exists an analytic function $f$ from $-F_{1}$ to $B$ such that $\|(T-z I) f(z)-x\|<\varepsilon$ for all $z$ in $-F_{1}$. Since $f$ is analytic on the neighborhood $-F_{1}$ of $U_{1} \cup C$, Runge's theorem for vector-valued analytic functions (whose proof is exactly the same as the proof of Runge's theorem in the scalar case) implies that $f$ can be uniformly approximated on $U_{1} \cup C$ by linear combinations of elementary elements of $V_{1}$. Therefore $f \in V$. Define $f_{1}$ in $V$ by

$$
f_{1}=-f+\alpha\left(x+\tau f, z_{0}, \cdot\right) .
$$

Then

$$
\tau f_{1}=-\tau f+\lim _{z \rightarrow \infty} z\left(z-z_{0}\right)^{-1}(x+\tau f)=x .
$$

Also,

$$
\begin{aligned}
& \left\|H f_{1}\right\|=\sup \left\{\left\|(T-z I) f_{1}(z)+\tau f_{1}\right\|: z \in U_{1}\right\} \\
& =\sup \left\{\left\|-(T-z I) f(z)+\left(z-z_{0}\right)^{-1}(T-z I)(x+\tau f)+\tau f_{1}\right\|: z \in U_{1}\right\} \\
& \leqq \sup \left\{\|(T-z I) f(z)-x\|: z \in U_{1}\right\} \\
& \quad \quad \quad \sup \left\{\left\|\left(z-z_{0}\right)^{-1}(T-z I)(x+\tau f)\right\|: z \in U_{1}\right\} \\
& \leqq \varepsilon+\sup \left\{\left\|\left(z-z_{0}\right)^{-1}(T-z I)(x+\tau f)\right\|: z \in U_{1}\right\} .
\end{aligned}
$$

Now

$$
\|x+\tau f\|=\lim _{z \rightarrow \infty}\|-[(T-z I) f(z)-x]\| \leqq \varepsilon .
$$

Thus

$$
\begin{aligned}
\left\|\left(z-z_{0}\right)^{-1}(T-z I)(x+\tau f)\right\| & \leqq\left\|\left[\left(z-z_{0}\right)^{-1}\left(T-z_{0} I\right)-I\right](x+\tau f)\right\| \\
& \leqq\left[c^{-1}\|T\|+c^{-1}\left|z_{0}\right|+1\right] \varepsilon .
\end{aligned}
$$

Thus

$$
\left\|H f_{1}\right\| \leqq\left[c^{-1}|| T \|+c^{-1}\left|z_{0}\right|+2\right] \varepsilon .
$$

Since $\varepsilon$ is arbitrary, $x \in N$. Thus $N\left(F_{1}, T\right) \subset N$.

We next show that $M\left(F_{2}, T^{*}\right) \subset M$, where $M$ is defined in the above corollary. It is sufficient to consider $u$ in $M\left(F_{2}, T^{*}\right)$ such that there exists an analytic function $g$ from $-F_{2}$ to $B^{*}$ with $\left(T^{*}-z I\right) g(z)=u$ 
for all $z$ in $-F_{2}$, since the set of such $u$ is dense in $M\left(F_{2}, T^{*}\right)$. Since $g$ is analytic on the neighborhood $-F_{2}$ of $U_{2} \cup C, g \in V_{2} \subset V^{*}$. By the definition of $H^{*}$ it follows that $H^{*} g=\tau^{*} u$. Thus $u \in M$, so that

$$
M\left(F_{2}, T^{*}\right) \subset M \text {. }
$$

Since $M^{\perp}=N$ by the above corollary, it follows that

$$
N\left(F_{1}, T\right)^{\perp} \supset M\left(F_{2}, T^{*}\right) .
$$

Since this holds for all operators $T$, we may replace $T$ by $T^{*}$ and interchange $F_{1}$ with $F_{2}$ to obtain

$$
N\left(F_{2}, T^{*}\right)^{\perp} \supset M\left(F_{1}, T\right) \text {, or } M\left(F_{1}, T\right)^{\perp} \supset N\left(F_{2}, T^{*}\right) .
$$

It remains to consider open sets $G_{1}$ and $G_{2}$ which cover the complex plane. There exists an unbounded simple open set $U_{1}$ such that $\bar{U}_{1} \subset G_{2} \cup-\sigma(T)$ and $\bar{U}_{2} \subset G_{1}$, where $U_{2}$ is the bounded simple open set which is complementary to $U_{1}$. Define $V, M$, and $N$ as in the above corollary. We first show $N \subset N\left(\bar{G}_{1}, T\right)$. Consider $x$ in $N$, so that for each $\varepsilon>0$ there exists $f$ in $V$ with $\tau f=x$ and $\|H f\|<\varepsilon$. This implies that

$$
\|(T-z I) f(z)+x\|=\|(T-z I)(-f(z))-x\|<\varepsilon
$$

for all $z$ in $U_{1}$. Since $-\bar{G}_{1} \subset U_{1}$, it follows that $x \in N\left(\bar{G}_{1}, T\right)$. We next show $M \subset M\left(\bar{G}_{2}, T\right)$. Consider $u$ in $M_{0}$, so that there exists $g$ in $V^{*}$ with $H^{*} g=\tau^{*} u$. Thus $\left(T^{*}-z I\right) g(z)=u$ for all $z$ in $U_{2}$. If $z \in-\sigma(T)$ let $g(z)=\left(T^{*}-z I\right)^{-1} u$. We thus obtain an analytic extension of $g$ to $U_{2} \cup-\sigma(T)$ which has the property that $\left(T^{*}-z I\right) g(z)=u$ for all $z$ in $U_{2} \cup-\sigma(T)$. Since $-\bar{G}_{2} \subset U_{2} \cup-\sigma(T)$, it follows that $u \in M\left(\bar{G}_{2}, T^{*}\right)$. Thus $M_{0} \subset M\left(\bar{G}_{2}, T^{*}\right)$, and therefore $M \subset M\left(\bar{G}_{2}, T^{*}\right)$. Since $M^{\perp}=N$ and is reflexive, $N^{\perp}=M$. Hence $N\left(\bar{G}_{1}, T\right)^{\perp} \subset M\left(\bar{G}_{2}, T^{*}\right)$. In this inclusion we may replace $T$ by $T^{*}$ and interchange $G_{1}$ and $G_{2}$ to obtain

$$
N\left(\bar{G}_{2}, T^{*}\right)^{\perp} \subset M\left(\bar{G}_{1}, T\right) \text { or } M\left(\overline{G_{1}}, T\right)^{\perp} \subset N\left(\bar{G}_{2}, T^{*}\right) .
$$

This completes the proof of Theorem 2.

We close this section by considering an example, which will show that $M(F, T) \neq N(F, T)$ in general and that not every operator admits a duality theory of type 1. Let $\left\{x_{n}\right\}_{n=-\infty}^{\infty}$ be a complete orthonormal basis for a Hilbert space $B$, and let $\left\{a_{n}\right\}_{n=-\infty}^{\infty}$ be a sequence of real numbers with $2^{-1}<a_{n}<2$ and

$$
\lim \sup _{n \rightarrow \infty}\left(a_{1} a_{2} \ldots a_{n}\right)^{1 / n}=\lim \sup _{n \rightarrow \infty}\left(a_{-1} a_{-2} \ldots a_{-n}\right)^{1 / n}=2
$$

and

$$
\lim \inf _{n \rightarrow \infty}\left(a_{1} a_{2} \ldots a_{n}\right)^{1 / n}=\liminf \underset{n \rightarrow \infty}{ }\left(a_{-1} a_{-2} \ldots a_{-n}\right)^{1 / n}=2^{-1} .
$$


Define the operator $T$ on $B$ by $T\left(\sum \alpha_{i} x_{i}\right)=\sum \alpha_{i} a_{i} x_{i+1}$. Then $T^{*}, T^{-1}$, and $\left(T^{*}\right)^{-1}$ are operators of the same type as $T$. For each $r$ with $2^{-1}<r<2$ let $K_{r}=\{z:|z| \leqq r\}$ and $L_{r}=\{z:|z| \geqq r\}$. We shall show that $M\left(K_{r}, T\right)=M\left(L_{r}, T\right)=\{0\}$, that $N\left(K_{r}, T\right)=N\left(L_{r}, T\right)=B$, and that corresponding equalities hold for $T^{*}$.

To show $M\left(K_{r}, T\right)=\{0\}$, let $x$ be any vector in $B$ such that there exists an analytic function $f$ from $-K_{r}$ to $B$ with $(T-z I) f(z)=x$ for all $z$ in $-K_{r}$. The Laurent expansion for $f$ is therefore

$$
f(z)=-\sum_{n=0}^{\infty} z^{-n-1} T^{n} x .
$$

Since this series converges for $|z|>r$ we have

$$
\text { lim } \sup _{n \rightarrow \infty}\left\|T^{n} x\right\|^{1 / n} \leqq r<2 .
$$

By the formula for $T$, this implies that $x=0$. Thus $M\left(K_{r}, T\right)=\{0\}$. Since $T^{-1}$ and $T^{*}$ are operators of the same type as $T$, we also have $M\left(K_{r}, T^{-1}\right)=M\left(K_{r}, T^{*}\right)=\{0\}$.

Now consider $x \in M\left(L_{r}, T\right)$. Thus there exists $f$ analytic for $|z|<r$ with values in $B$ such that $(T-z I) f(z)=x$. Therefore

$$
\left(z^{-1} I-T^{-1}\right)(z f(z))=T^{-1} x
$$

for $|z|<r$. Thus $-z f(z)=g\left(z^{-1}\right)$ is an analytic function of $z^{-1}$ for $\left|z^{-1}\right|>r^{-1}$ and $\left(T^{-1}-z^{-1} I\right) g\left(z^{-1}\right)=T^{-1} x$. Therefore $T^{-1} x \in M\left(K_{r^{-1}}, T^{-1}\right)$. By the above, this implies that $T^{-1} x=0$. Therefore $x=0$. Therefore $M\left(L_{r}, T\right)=\{0\}$. Similarly, $M\left(L_{r}, T^{*}\right)=\{0\}$. This already shows that $T$ does not admit a duality theory of type 1 , since by the above it is not true that

$$
M\left(K_{r}, T\right)^{\perp} \subset M\left(L_{s}, T^{*}\right) \text { if } 2^{-1}<s<r<2 .
$$

To show that $N\left(K_{r}, T\right)=B$, choose $s$ with $2^{-1}<s<r<2$. Then $N\left(K_{r}, T\right)^{\perp} \subset M\left(L_{s}, T^{*}\right)=\{0\}$ since $T$ admits a duality theory of type 4. It follows that $N\left(K_{r}, T\right)=B$. The equality $N\left(L_{r}, T\right)=B$ is proved similarly.

\section{The other duality theories.}

Definition 7. The operator $T$ on the reflexive Banach space $B$ is said to satisfy condition $\alpha$ if $N\left(F_{1}, T\right) \subset M\left(F_{2}, T\right)$ whenever $F_{1}$ and $F_{2}$ are closed and $F_{1} \subset$ interior $F_{2}$.

THEOREM 3. An operator $T$ which satisfies condition $\alpha$ admits a duality theory of type 1.

Proof. Assume that $T$ satisfies condition $\alpha$. Let $F_{1}$ and $F_{2}$ be disjoint compact sets. Then 


$$
M\left(F_{1}, T\right) \subset N\left(F_{1}, T\right) \text {, so that } M\left(F_{1}, T\right)^{\perp} \supset N\left(F_{1}, T\right)^{\perp} \supset M\left(F_{2}, T^{*}\right)
$$

by Theorem 2 .

Consider now open sets $G_{1}$ and $G_{2}$ which cover the complex plane. Choose an open set $G_{3}$ such that $\bar{G}_{3} \subset G_{1}$ and such that $G_{2}$ and $G_{3}$ cover the complex plane. Then $N\left(\bar{G}_{3}, T\right) \subset M\left(\bar{G}_{1}, T\right)$ by condition $\alpha$. Thus $M\left(\overline{G_{1}}, T\right)^{\perp} \subset N\left(\bar{G}_{3}, T\right)^{\perp} \subset M\left(\bar{G}_{2}, T^{*}\right)$ by Theorem 2 . This completes the proof of Theorem 3 .

We now investigate conditions which are sufficient for $T$ to admit duality theories of types 2 and 3 .

Definition 8. The operator $T$ on the reflexive Banach space $B$ satisfies condition $\beta$ if for every open set $U$ and every sequence $\left\{f_{n}\right\}$ of analytic functions from $U$ to $B$ such that $(T-z I) f_{n}(z) \rightarrow 0$ uniformly on $U$ as $n \rightarrow \infty$ it follows that the sequence $\left\{f_{n}\right\}$ is uniformly bounded on compact subsets of $U$.

There are various reformulations of Definition 8 . We shall need the following: $T$ satisfies condition $\beta$ if and only if for every open set $U$ and every sequence $\left\{f_{n}\right\}$ of analytic functions from $U$ to $B$ and every $x$ in $B$ such that $(T-z I) f_{n}(z) \rightarrow x$ uniformly on compact subsets of $U$ as $n \rightarrow \infty$ it follows that $\left\{f_{n}\right\}$ is uniformly bounded on compact subsets of $U$. That this reformulation implies condition $\beta$ is clear. To see that $\beta$ implies this reformulation, let $\left\{f_{n}\right\}$ be a sequence of analytic functions from $U$ to $B$ such that $(T-z I) f_{n}(z) \rightarrow x$ uniformly on compact subsets of $U$, and let $K$ be a compact subset of $U$. Then there exists a neighborhood $U_{1}$ of $K$ such that $(T-z I) f_{n}(z) \rightarrow x$ uniformly on $U_{1}$. If $\left\{f_{n}\right\}$ is not uniformly bounded on $K$, by passing to a subsequence if necessary we may assume that $d_{n+1}>n+d_{n}$ for each $n$, where

$$
d_{n}=\sup \left\{\left\|f_{n}(z)\right\|: z \in K\right\} .
$$

Thus the sequence $\left\{g_{n}\right\}$ defined by $g_{n}=f_{n+1}-f_{n}$ is not uniformly bounded on $K$. On the other hand,

$$
(T-z I) g_{n}(z)=(T-z I) f_{n+1}(z)-(T-z I) f_{n}(z)
$$

converges uniformly to $x-x=0$ on $U_{1}$. This contradicts condition $\beta$. Therefore $\left\{f_{n}\right\}$ is uniformly bounded on $K$, as was to be proved.

Definition 9. The operator $T$ on the reflexive Banach space $B$ will be said to satisfy condition $\gamma$ if for every open set $U$ and every non-zero analytic $f$ from $U$ to $B$ there exists $z$ in $U$ with $(T-z I) f(z) \neq 0$.

THEOREM 4. If $T$ satisfies condition $\beta$ and if $F$ is an arbitrary closed subset of the complex plane, then (1) $M(F, T)=N(F, T)$, (2) for 
each $x$ in $M(F, T)$ there exists an analytic function $f$ from $-F$ to $B$ with $(T-z I) f(z)=x$ for all $z$ in $-F$, and (3) $T$ satisfies conditions $\alpha$ and $\gamma$.

Proof. For each $x$ in $N(F, T)$ there exists a sequence $\left\{f_{n}\right\}$ of analytic functions from $-F$ to $B$ such that $\left\|(T-z I) f_{n}(z)-x\right\|<n^{-1}$ for all $z$ in $-F$. By condition $\beta,\left\{f_{n}\right\}$ is uniformly bounded on compact subsets of $-F$, so that there exists a subset, which we continue to call $\left\{f_{n}\right\}$, converging pointwise in the weak topology of $B$ to an analytic function $f$ from $-F$ to $B$. Clearly $(T-z I) f(z)=x$ for all $z$ in $-F$. This proves assertions (1) and (2).

If $F_{1}$ and $F_{2}$ are closed and $F_{1} \subset$ interior $F_{2}$, then $N\left(F_{1}, T\right)=$ $M\left(F_{1}, T\right) \subset M\left(F_{2}, T\right)$ by (1), so that $T$ satisfies condition $\alpha$.

If $T$ does not satisfy condition $\gamma$, there exists an open set $U$ and a non-zero analytic function $f$ from $U$ to $B$ with $(T-z I) f(z)=0$ for all $z$ in $U$. Let $f_{n}=n f$, so that $(T-z I) f_{n}(z) \rightarrow 0$ as $n \rightarrow \infty$ uniformly on $U$. Since $T$ satisfies condition $\beta$, it follows that the sequence $\{n f\}$ is pointwise bounded. This contradiction proves that $T$ satisfies condition $\gamma$.

The theorem just proved is related in part to results of Wermer [5]. As a corollary of this theorem, $T$ admits a duality theory of type 1 if $T$ satisfies condition $\beta$.

THeorem 5. If $T^{*}$ satisfies condition $\beta$, then $T$ admits a duality theory of type 2. If $T$ and $T^{*}$ satisfy condition $\beta$, then $T$ admits a duality theory of type 3.

Proof. Assume that $T^{*}$ satisfies condition $\beta$. Then $T^{*}$, and therefore $T$, admits a duality theory of type 1 . Also, $T^{*}$ satisfies condition $\gamma$. Let $G_{1}, \cdots, G_{n}$ be open sets covering the complex plane. If $M\left(\bar{G}_{1}, T\right), \cdots, M\left(\bar{G}_{n}, T\right)$ do not $\operatorname{span} B$, there exists $u \neq 0$ in $B^{*}$ orthogonal to these subspaces. Since $G_{1}, \cdots, G_{n}$ cover the complex plane, $\bigcap_{i=1}^{n}-G_{i}$ is void. There therefore exist open sets $U_{1}, \cdots, U_{n}$ such that $-G_{i} \subset U_{i}$ and $\bigcap_{i=1}^{n} \bar{U}_{i}$ is void. Since $U_{i}$ and $G_{i}$ cover the complex plane and since $T$ admits a duality theory of type 1 , we see that

$$
M\left(\bar{G}_{i}, T\right)^{\perp} \subset M\left(\bar{U}_{i}, T^{*}\right) .
$$

Thus $u \in M\left(\bar{U}_{i}, T^{*}\right)$ for each $i$. Since $T^{*}$ satisfies condition $\beta$ it follows from Theorem 4 that there exist an analytic function $g_{i}$ from $-\bar{U}_{i}$ to $B^{*}$ with $\left(T^{*}-z I\right) g_{i}(z)=u$ for all $z$ in $-\bar{U}_{i}$. If $z \in-\bar{U}_{i} \cap-\bar{U}_{j}$, we have $\left(T^{*}-z I\right)\left(g_{i}(z)-g_{j}(z)\right)=0$. Since $g_{i}-g_{j}$ is analytic on $-\bar{U}_{i} \cap-\bar{U}_{j}$ and since $T^{*}$ satisfies condition $\gamma$, it follows that $g_{i}(z)=g_{j}(z)$ for $z$ in $-\bar{U}_{i} \cap-\bar{U}_{j}$. Thus if we define the function $g$ by $g(z)=g_{i}(z)$ for $z$ in $-\bar{U}_{i}$, then $g$ is uniquely defined and is analytic on $\bigcup_{i=1}^{n}-\bar{U}_{i}$. Also 
$\left(T^{*}-z I\right) g(z)=u$ whenever $g$ is defined. But the sets $-\bar{U}_{i}$ cover the plane because $\bigcap_{i=1}^{n} \bar{U}_{i}$ is void. Also $g(z)=\left(T^{*}-z I\right)^{-1} u \rightarrow 0$ as $|z| \rightarrow \infty$. Thus $g$ is everywhere analytic and vanishes at infinity, so that $g=0$. Thus $u=\left(T^{*}-z I\right) g(z)=0$. This contradiction implies that $M\left(\overline{G_{1}}, T\right)$, $\cdots, M\left(\bar{G}_{n}, T\right)$ span $B$. Therefore $T$ admits a duality theory of type 2 .

Assume now that $T$ also satisfies condition $\beta$. We must show that $T$ admits a duality theory of type 3 . First consider a bounded open set $H$ such that $-\bar{H}$ is connected. By Theorem 4 for each $x$ in $M=M(\bar{H}, T)$ there exists an analytic function $f(x, \cdot)$ from $U=-\bar{H}$ to $B$ such that $(T-z I) f(x, z)=x$ for all $z$ in $U$. Since $T$ satisfies condition $\beta$, and therefore condition $\gamma$, the function $f(x, \cdot)$ is unique. For each $z_{0}$ in $U$ define the linear transformation $T_{z_{0}}$ from $M$ to $B$ by $T_{z_{0}} x=f\left(x, z_{0}\right)$. It follows that $\left(T-z_{0} I\right) T_{z_{0}}$ is the identity operator on $M$. Assume that there exists $z_{0}$ in $U$ such that $T_{z_{0}}$ is an unbounded transformation. There therefore exists a sequence $\left\{x_{n}\right\}$ from $M$ such that $x_{n} \rightarrow 0$ and $\left\|T_{z_{0}} x_{n}\right\| \rightarrow \infty$ as $n \rightarrow \infty$. Since $T$ satisfies condition $\beta$, it follows that $\left\{f\left(x_{n}, z_{0}\right)\right\}=\left\{T_{z_{0}} x_{n}\right\}$ is bounded. This contradiction implies that $T_{z_{0}}$ is a bounded transformation for each $z_{0}$ in $U$. If $z$ in $U$ is sufficiently large and if $x \in M, T_{z} x=(T-z I)^{-1} x \in M$. Since $T_{z} x$ is an analytic function on $U$ and since $U$ is connected, it follows that $T_{z} x \in M$ for all $x$ in $M$ and all $z$ in $U$. Thus for each $z$ in $U$ the operator $T_{z}$ on $M$ is a right inverse of $(T-z I) / M$. To show that $T_{z}$ is the inverse of $(T-z I) / M$ it is sufficient to establish that $x=0$ whenever $x \in M$ and $(T-z I) x=0$. To this end, consider $x$ in $M$ with $(T-z I) x=0$. Define the analytic function $h$ from $U$ to $B$ by $h(\lambda)=x+(\lambda-z) T_{\lambda} x$. Then

$$
\begin{aligned}
(T-\lambda I) h(\lambda) & =(T-z I) x+(z-\lambda) x+(\lambda-z)(T-\lambda I) T_{\lambda} x \\
& =0+(z-\lambda) x+(\lambda-z) x=0 .
\end{aligned}
$$

Since $T$ satisfies condition $\beta$, and therefore condition $\gamma$, this implies $h=0$. Thus $x=h(z)=0$. Therefore $(T-z I) / M$ has an inverse for all $z$ in $U$, so that $\sigma(T / M(\bar{H}, T)) \subset \bar{H}$.

Now let a covering $G_{1}, \cdots, G_{n}$ of the complex plane by open sets be given. There exists a family $\left\{H_{i k}\right\}, 1 \leqq i \leqq n, 1 \leqq k \leqq m_{i}$, of open sets which cover the complex plane such that $H_{i k} \subset G_{i}$ and either $H_{i k}$ is bounded and $-\bar{H}_{i k}$ is connected or $\bar{H}_{i k} \subset-\sigma(T)$. From the preceding paragraphs we see that the $M\left(\bar{H}_{i k}, T\right)$ span $B$ and that

$$
\sigma\left(T / M\left(\bar{H}_{i k}, T\right)\right) \subset \bar{H}_{i k}
$$

if $H_{i k}$ is bounded and $-\bar{H}_{i k}$ is connected. Also $\sigma\left(T / M\left(\bar{H}_{i k}, T\right)\right) \subset \bar{H}_{i k}$ if $\bar{H}_{i k} \subset-\sigma(T)$ because $M\left(\bar{H}_{i k}, T\right)$ consists only of the zero vector in this case. If this were not true, there would exist $x \neq 0$ in $M\left(\bar{H}_{i k}, T\right)$ and 
an analytic function $f$ from $-\bar{H}_{i k}$ to $B$ such that $(T-z I) f(z)=x$. If we extended the definition of $f$ to $-\sigma(T)$ by defining $f(z)=(T-z I)^{-1} x$ for $z$ in $-\sigma(T)$, then $f$ becomes an analytic function defined everywhere and vanishing at infinity. Thus $f=0$. Therefore $x=(T-z I) f=0$. Hence $\sigma\left(T / M\left(\bar{H}_{i k}, T\right)\right) \subset \bar{H}_{i k}$ for all $i$ and $k$. Define $M_{i}$ to be the subspace spanned by the sets $M\left(\bar{H}_{i 1}, T\right), \cdots, M\left(\bar{H}_{i m_{i}}, T\right)$. Since

$$
M\left(\bar{H}_{i k}, T\right) \subset M\left(\bar{G}_{i}, T\right)
$$

for each $k$, we have $M_{i} \subset M\left(\overline{G_{i}}, T\right)$. Also $M_{1}, \cdots, M_{n}$ span $B$. To complete the proof of the theorem we must show that $\sigma\left(T / M_{i}\right) \subset \overline{G_{i}}$. As before, with the set $H$ replaced by $G_{i}$, for each $x$ in $M_{i} \subset M\left(\overline{G_{i}}, T\right)$ there exists a unique analytic function $f(x, \cdot)$ from $-\overline{G_{i}}$ to $B$ such that $(T-z I) f(x, z)=x$ for all $z$ in $-\bar{G}_{i}$. As before, for each $z$ in $-\bar{G}_{i}$ define the linear transformation $T_{z}$ from $M_{i}$ to $B$ by $T_{z} x=f(x, z)$. As before, $T_{z}$ is a bounded linear transformation for each $z$ in $-\bar{G}_{i}$. If the set $H_{i k}$ is bounded and $-\bar{H}_{i k}$ is connected, we see as before that the spaces $M\left(\bar{H}_{i k}, T\right)$ are invariant under $T_{z}$ for each $z$ in $-\bar{H}_{i k} \supset-\bar{G}_{i}$. Since otherwise $H_{i k}$ has the property that $M\left(\bar{H}_{i k}, T\right)$ consists only of the vector 0 , and since the $M\left(\bar{H}_{i k}, T\right)$ span $M$, it follows that $M_{i}$ is invariant under $T_{z}$ for all $z$ in $-\overline{G_{i}}$. Thus the operator $T_{z}$ on $M_{i}$ is a right inverse of $(T-z I) / M_{i}$. As before, it follows that $T_{z}$ is the inverse of $(T-z I) / M_{i}$ for each $z$ in $-\bar{G}_{i}$. Thus $\sigma\left(T / M_{i}\right) \subset \bar{G}_{i}$, as was to be proved.

As an immediate consequence of the preceding theorems, it follows that $T$ and $T^{*}$ both admit duality theories of types $1,2,3$, and 4 whenever $T$ and $T^{*}$ both satisfy condition $\beta$.

Definition 10. Let $r$ be a function from the complex plane to the set $[0, \infty]$. The function $r$ will be said to be a modulus of control for analytic functions if for every open set $U$ and every sequence $\left\{f_{n}\right\}$ of complex-valued analytic functions on $U$ such that $\left|f_{n}(z)\right| \leqq r(z)$ for all $z$ in $U$ the sequence $\left\{f_{n}\right\}$ is uniformly bounded on all compact subsets of $U$.

THEOREM 6. Let $T$ be an operator on a reflexive Banach space $B$. Let the function $r$ defined by $r(z)=\left\|(T-z I)^{-1}\right\|$ if $z \in-\sigma(T)$ and $r(z)=\infty$ if $z \in \sigma(T)$ be a modulus of control for analytic functions. Then both $T$ and $T^{*}$ satisfy condition $\beta$ and therefore admit duality theories of types 1, 2, 3, and 4 .

Proof. Since $\left\|(T-z I)^{-1}\right\|=\left\|\left(T^{*}-z I\right)^{-1}\right\|$ whenever $z \in-\sigma(T)$, it is sufficient to show that $T$ satisfies condition $\beta$. To do this, let $U$ be open and let $\left\{f_{n}\right\}$ be a sequence of analytic functions from $U$ to $B$ such 
that $(T-z I) f_{n}(z) \rightarrow 0$ uniformly on $U$ as $n \rightarrow \infty$. It is then possible to choose a constant $K$ such that $\left\|f_{n}(z)\right\| \leqq K\left\|(T-z I)^{-1}\right\|$ for all $n$ and all $z$ in $U$. Assume that there exists a compact subset $C$ of $U$ on which $\left\{f_{n}\right\}$ is not uniformly bounded. By the uniform boundedness theorem [3], it is possible to choose $u$ in $B^{*}$ such that the sequence $\left\{g_{n}\right\}$ of complex-valued analytic function on $U$ defined by $g_{n}(z)=\left\langle f_{n}(z), u\right\rangle$ is not uniformly bounded on $C$. Since $\left|g_{n}(z)\right| \leqq K\|u\| r(z)$ for all $z$ in $U$ and since $r$ is a modulus of control for analytic functions, it follows that $\left\{g_{n}\right\}$ is uniformly bounded on $C$. This contradiction shows that $\left\{f_{n}\right\}$ is uniformly bounded on compact subsets of $U$, so that $T$ satisfies condition $\beta$, as was to be proved.

The following corollary is closely related to and was suggested by an unpublished result due to F. Wolf.

COROLLARY. If $T$ is an operator on a reflexive Banach space such that $\sigma(T)$ is a subset of the real line and such that

$$
\int \log ^{+} \log ^{+} \sup \left\{\left\|(T-z I)^{-1}\right\|: I(z)=y\right\} d y
$$

is finite, then $T$ admits duality theories of types 1,2,3, and 4.

Proof. By Wolf [6], the function $r$ defined by $r(z)=\left\|(T-z I)^{-1}\right\|$ is a modulus of control for analytic functions, so that the result follows from Theorem 6 .

It follows that a Hermitian operator, for instance, admits duality theories of types 1, 2, 3, and 4. From this fact it is not difficult to derive the spectral theorem.

\section{REFERENCES}

1. E. Bishop, Speciral theory for operators on a Banach Space, Trans. Amer. Math. Soc., 86 (1957), 414-445.

2. N. Dunford, Spectral operators, Pacific J. Math., 4 (1954), 321-354.

3. E. Hille, Functional analysis and semi-groups, Amer. Math. Soc. Colloquium Publications, 31 (1948).

4. E. R. Lorch, The iniegral representational of weakly almost periodic transformations in reflexive vector spaces, Trans. Amer. Math. Soc., 49 (1941), 18-40.

5. J. Wermer, The existence of invariant subspaces, Duke Math. J., 19 (1952), 615-622.

6. F. Wolf, On majorants of subharmonic and analytic functions, Bull. Amer. Math. Soc., 48 (1942), 925-932.

7. - Operators in a Banach space which admit a generalized spectral decomposition, Nederl. Akad. Wetensch. Indag. Math., 19 (1957), 302-311.

UNIVERSity of CALIForNia BERKELEY 
